\title{
ON GROUPS RELATED TO THE HECKE GROUPS
}

\author{
MARVIN I. KNOPP AND MORRIS NEWMAN
}

(Communicated by Dennis A. Hejhal)

\begin{abstract}
Let $\left[\begin{array}{cc}1 & \lambda_{1} \\ 0 & 1\end{array}\right],\left[\begin{array}{cc}1 & 0 \\ \lambda_{2} & 1\end{array}\right]$ be parabolic elements of $\operatorname{PSL}(2, R)$, where $\lambda_{1}, \lambda_{2}>0$. The principal result shown here is that $K\left(\lambda_{1}, \lambda_{2}\right)$, the group generated by these elements, is discrete if and only if $\lambda_{1} \lambda_{2} \geq 4$, or $\lambda_{1} \lambda_{2}=$ $4 \cos ^{2}(\pi / p)$, where $p$ is an integer $\geq 3$. When $\lambda_{1} \lambda_{2}=4 \cos ^{2}(\pi / p), K\left(\lambda_{1}, \lambda_{2}\right)$ is conjugate to the classical Hecke group $H(2 \cos (\pi / p))$ if $p$ is odd; while if $p$ is even, $K\left(\lambda_{1}, \lambda_{2}\right)$ is conjugate to a subgroup of $H(2 \cos (\pi / p))$ of index 2. When $\lambda_{1} \lambda_{2} \geq 4, K\left(\lambda_{1}, \lambda_{2}\right)$ is conjugate to a subgroup of $H\left(\sqrt{\left(\lambda_{1} \lambda_{2}\right)}\right)$ of index 2 . In all of these cases $K\left(\lambda_{1}, \lambda_{2}\right)$ is the free product of two cyclic groups.
\end{abstract}

This article has its genesis in [3], a continuing investigation of some littlestudied aspects of the Hecke correspondence between modular forms, on the one hand, and Dirichlet series with functional equations, on the other. To describe the questions treated in [3], consider the ordinary Dirichlet series

$$
\phi(s)=\sum_{n=1}^{\infty} a_{n} n^{-s}, \quad \psi(s)=\sum_{n=1}^{\infty} b_{n} n^{-s},
$$

where the coefficients have at most polynomial growth in $n$, so that both series converge in a right half-plane. Assume as well that $\phi(s)$ and $\psi(s)$ can be continued meromorphically into the entire $s$-plane and satisfy a general functional equation of the "Hecke type",

$$
\left(2 \pi / \lambda_{1}\right)^{-s} \Gamma(s) \phi(s)=C\left(2 \pi / \lambda_{2}\right)^{-(k-s)} \Gamma(k-s) \psi(k-s) ;
$$

here $\Gamma(s)$ is the gamma-function, $k$ is a rational integer, $\lambda_{1}$ and $\lambda_{2}$ are positive numbers, and $C$ is complex. (See $[1,2,4]$.)

The questions considered in [3] include: (i) which pairs $\lambda_{1}, \lambda_{2}$ admit nontrivial solutions $\phi(s), \psi(s)$ of (1)?; (ii) with fixed $\lambda_{1}$ and $\lambda_{2}$, to what extent can $\phi(s)$ and $\psi(s)$ differ? An illustration of the results contained in [3] is: if $\lambda_{1}=\lambda_{2}=1$ in (1), then $\phi(s)=C \psi(s)$. As one might guess, the results of [3] arise from a consideration of the inverse Mellin transforms of $\left(2 \pi / \lambda_{1}\right)^{-s} \Gamma(s) \phi(s)$ and $\left(2 \pi / \lambda_{2}\right)^{-s} \Gamma(s) \psi(s)$. This brings into play modular functions with respect to certain groups of real linear fractional transformations acting on the upper half-plane. In [3] the problem arises naturally to determine when these groups are discrete; this will be the problem treated here.

Received by the editors January 31, 1992.

1991 Mathematics Subject Classification. Primary 11F06, 20H10.

The first author's research was supported in part by NSA/MSP Grant MDA90-H-4025.

(c) 1993 American Mathematical Society $0002-9939 / 93 \$ 1.00+\$ .25$ per page 
We now describe these groups. Set

$$
T=\left[\begin{array}{cc}
0 & 1 \\
-1 & 0
\end{array}\right], \quad S(\lambda)=\left[\begin{array}{ll}
1 & \lambda \\
0 & 1
\end{array}\right], \quad W(\lambda)=\left[\begin{array}{ll}
1 & 0 \\
\lambda & 1
\end{array}\right],
$$

regarded as elements of $\operatorname{PSL}(2, R)$. Suppose that $\lambda_{1}, \lambda_{2}>0$, and put

$$
K\left(\lambda_{1}, \lambda_{2}\right)=\left\{S\left(\lambda_{1}\right), W\left(\lambda_{2}\right)\right\}
$$

the group generated by $S\left(\lambda_{1}\right)=\left[\begin{array}{cc}1 & \lambda_{1} \\ 0 & 1\end{array}\right]$, and $W\left(\lambda_{2}\right)=\left[\begin{array}{ll}1 & 0 \\ \lambda_{2} & 1\end{array}\right]$. We also set

$$
K(\lambda)=\{S(\lambda), W(\lambda)\}, \quad H(\lambda)=\{T, S(\lambda)\} .
$$

Then $H(\lambda)$ is a Hecke group, $K(\lambda)$ is a subgroup of $H(\lambda)$, and $K(\lambda)=$ $K(\lambda, \lambda)$.

Although we are primarily interested in $K\left(\lambda_{1}, \lambda_{2}\right)$, we will work instead with the group $K(\lambda)$ and then carry over the results to $K\left(\lambda_{1}, \lambda_{2}\right)$. This procedure is justified by the following lemma:

Lemma 1. Suppose that $\lambda_{1}, \lambda_{2}, \mu_{1}, \mu_{2}>0, \lambda_{1} \lambda_{2}=\mu_{1} \mu_{2}$. Then the groups $K\left(\lambda_{1}, \lambda_{2}\right), K\left(\mu_{1}, \mu_{2}\right)$ are conjugate.

Proof. Set $\alpha=\sqrt{\left(\mu_{1} / \lambda_{1}\right)}=\sqrt{\left(\lambda_{2} / \mu_{2}\right)}, D=\left[\begin{array}{cc}\alpha & 0 \\ 0 & 1 / \alpha\end{array}\right]$. Then a brief calculation shows that

$$
D S\left(\lambda_{1}\right) D^{-1}=S\left(\mu_{1}\right), \quad D W\left(\lambda_{2}\right) D^{-1}=W\left(\mu_{2}\right),
$$

so that $D K\left(\lambda_{1}, \lambda_{2}\right) D^{-1}=K\left(\mu_{1}, \mu_{2}\right)$. This completes the proof.

Thus by choosing $\mu_{1}=\mu_{2}=\sqrt{\left(\lambda_{1} \lambda_{2}\right)}$, we find that $K\left(\lambda_{1}, \lambda_{2}\right)$ and $K(\lambda)$, $\lambda=\sqrt{\left(\lambda_{1} \lambda_{2}\right)}$, are conjugate.

Our ultimate objective will be to prove the following:

Theorem 1. The group $K\left(\lambda_{1}, \lambda_{2}\right)$ is discrete if and only if $\lambda_{1} \lambda_{2} \geq 4$, or $\lambda_{1} \lambda_{2}=$ $4 \cos ^{2}(\pi / p)$, where $p$ is an integer $\geq 3 . K\left(\lambda_{1}, \lambda_{2}\right)$ is then structured as follows:

Case 1. $\lambda_{1} \lambda_{2} \geq 4$. Then $K\left(\lambda_{1}, \lambda_{2}\right)$ is conjugate to a subgroup of index 2 of the Hecke group $H(\lambda), \lambda=\sqrt{\left(\lambda_{1} \lambda_{2}\right)}$.

Case 2. $\lambda_{1} \lambda_{2}=4 \cos ^{2}(\pi / p), p$ even. Then $K\left(\lambda_{1}, \lambda_{2}\right)$ is conjugate to a subgroup of index 2 of the Hecke group $H(\lambda), \lambda=2 \cos (\pi / p)$.

Case 3. $\lambda_{1} \lambda_{2}=4 \cos ^{2}(\pi / p), p$ odd. Then $K\left(\lambda_{1}, \lambda_{2}\right)$ is conjugate to the Hecke group $H(2 \cos (\pi / p))$ and so is the free product of a cyclic group of order 2 and a cyclic group of order $p$.

We first prove some purely group-theoretic results.

Theorem 2. Let $H=\{x, y\}$ be a group generated by elements $x, y$, where $x^{2}=1$. Let $K=\{x y x, y\}$. Then $K$ is a normal subgroup of $H,(H: K)=1$ or 2 , and $H=K$ if and only if $x$ belongs to $K$. If $x$ does not belong to $K$, then $H=K+x K$.

Proof. We have

and

$$
x K x^{-1}=\{y, x y x\}=K, \quad \text { since } x^{2}=1
$$

$$
y K y^{-1}=K, \quad \text { since } y \text { belongs to } K \text {. }
$$

Hence $K$ is a normal subgroup of $H$. Thus $H / K$ is the group generated by $x, y$ with the relations $x^{2}=1, x y x=1, y=1$; and these imply that $H / K$ is of order 1 if $x$ belongs to $K, 2$ otherwise. This completes the proof.

We can determine exactly when $H=K$, if $H$ is a free product. 
Theorem 3. Put $u=x y$, and suppose in addition that $H=\{x\} *\{u\}$, the free product of the cyclic group $\{x\}$ of order 2 and the cyclic group $\{u\}$. Then $H=K$ if $\{u\}$ is finite and of odd order and $(H: K)=2, H=K+x K$, if $\{u\}$ is finite and of even order, or if $\{u\}$ is infinite.

Proof. Note first that $H=\{x, u\}$ and that $K=\{u x, x u\}=\left\{x u, u^{2}\right\}$.

Suppose first that $\{u\}$ is of order $p$, where $p$ is odd. Then $u^{2}$ belongs to $K, u^{p}$ belongs to $K$, so $u$ belongs to $K$. This in turn implies that $x$ belongs to $K$, so $K=H$.

Next, suppose that $\{u\}$ is of order $p$, where $p$ is even. Then if $x$ belongs to $K$, there are integers $a_{1}, b_{1}, a_{2}, b_{2}, \ldots$ such that

$$
x=(x u)^{a_{1}}\left(u^{2}\right)^{b_{1}}(x u)^{a_{2}}\left(u^{2}\right)^{b_{2}} \cdots .
$$

The exponent sums of $x$ modulo 2 and $u$ modulo $p$ on each side of this equation must agree, since $H=\{x\} *\{u\}$; so

$$
\begin{aligned}
& a_{1}+a_{2}+\cdots \text { is odd, } \\
& a_{1}+a_{2}+\cdots+2 b_{1}+2 b_{2}+\cdots \equiv 0 \bmod p .
\end{aligned}
$$

This is a contradiction, however, since $p$ is even, and so $x$ does not belong to $K$. Thus (by Theorem 2), $(H: K)=2, H=K+x K$.

Finally, suppose that $\{u\}$ is of infinite order so that $x^{2}=1$ is the only relation. Then as before, if $x$ belongs to $K$, equation (2) implies that

$$
\begin{aligned}
& a_{1}+a_{2}+\cdots \text { is odd, } \\
& a_{1}+a_{2}+\cdots+2 b_{1}+2 b_{2}+\cdots=0 .
\end{aligned}
$$

But this is also a contradiction, and so $x$ does not belong to $K$. Thus $(H: K)$ $=2, H=K+x K$. This completes the proof.

Proof of Theorem 1. We set up the correspondence

$$
x \rightarrow T, \quad y \rightarrow S(\lambda)
$$

so that

$$
x y x \rightarrow W(\lambda)^{-1}, \quad H \rightarrow H(\lambda), \quad K \rightarrow K(\lambda) .
$$

It is classical that the Hecke group $H(\lambda)$ is discrete if and only if $\lambda \geq 2$ or $\lambda=2 \cos (\pi / p)$, where $p$ is an integer $\geq 3$. It is also clear that if $F, G$ are arbitrary subgroups of $\operatorname{PSL}(2, R)$ such that $F$ is discrete and $G \subset F$, then $G$ is also discrete. These remarks, together with Lemma 1 and Theorems 2 and 3, constitute the proof of Theorem 1.

Some final remarks:

(a) It is known that $K\left(\lambda_{1}, \lambda_{2}\right)$ is free when $\lambda_{1} \lambda_{2} \geq 4$.

(b) $K\left(\lambda_{1}, \lambda_{2}\right)$ and the Hecke group $H(\lambda), \lambda=\sqrt{\left(\lambda_{1} \lambda_{2}\right)}$, are of the same "kind".

(c) $K\left(\lambda_{1}, \lambda_{2}\right)$ is always the free product of two cyclic groups.

\section{REFERENCES}

1. S. Bochner, Some properties of modular relations, Ann. of Math. (2) 53 (1951), 332-363.

2. K. Chandrasekharan and R. Narasimhan, Hecke's functional equation and arithmetical identities, Ann. of Math. (2) 74 (1961), 1-23. 
3. M. I. Knopp, Results related to Hamburger's theorem (in preparation).

4. C. L. Siegel, Bemerkungen zu einem Satz von Hamburger ueber die Funktionalgleichung der Riemannschen Zetafunktion, Math. Ann. 86 (1922), 276-279.

Department of Mathematics, Temple University, Philadelphia, Pennsylvania 19122

Department of Mathematics, University of California, Santa Barbara, California 93106 